\title{
The Numerical Integration of Two-Point Boundary Value Problems
}

1. Introduction. A general system of linear, ordinary, differential equations may be written, in matrix notation

$$
\dot{Y}=A(t) Y+F(t)
$$

where the dot denotes differentiation with respect to the independent variable, $t ; Y$ is the column matrix of unknowns,

$$
\left[\begin{array}{c}
y_{1} \\
\cdot \\
\cdot \\
y_{n}
\end{array}\right),
$$

$A(t)$ is a square matrix, and $F(t)$, the forcing functions, is another column matrix,

$$
\left(\begin{array}{l}
f_{1} \\
\cdot \\
\cdot \\
f_{n}
\end{array}\right) .
$$

Suppose that $r$ values $y_{1}(0) \cdots y_{r}(0)$ are given and at $t=T,(n-r)$ of the $y_{i}$ 's are given, viz. : $y_{i_{k}}, k=1, \cdots,(n-r)$. In order to solve (1) using high speed computing machinery, whether analogue or digital, it is essential to have $n$ conditions at one end of the interval $0-T$. One possibility is to carry the constants $y_{r+1}(0) \cdots y_{n}(0)$ along as unknowns during the integration, and then to determine them when the far end is reached. Another possibility consists of guessing $y_{r+1}(0) \cdots y_{n}(0)$ and then adjusting them. These techniques have been described by Tifford [1]. The first of these methods is awkward especially if a large number of unknowns must be carried; the second requires more integrations than the methods described below.

This paper is concerned with methods for converting a two-point boundary value problem into an initial value problem. For linear systems the conversion is accomplished with a finite number of integrations. In section 2, where nonlinear systems are considered, an iterative procedure is required.

2. Linear Systems-Method of Adjoint Equations. The adjoint system was developed by Bliss [2]; more recent developments are described by Wright [3]. The system adjoint to (1) is, by definition,

$$
-\dot{X}=\bar{A}(t) X,
$$


where the bar denotes the transposed matrix and $X$ is a column matrix

The $x_{i}^{\prime}$ 's and $y_{i}^{\prime}$ 's are related by

$$
\left(\begin{array}{l}
x_{1} \\
\cdot \\
\cdot \\
\cdot \\
x_{n}
\end{array}\right) .
$$

$$
\frac{d}{d t}\left(x_{i} y_{i}\right)=x_{i} f_{i},
$$

where the repeated suffix implies summation from 1 to $n$. A proof of (3) follows:

Consider

$$
\frac{d}{d t}\left(x_{i} y_{i}\right) \equiv \frac{d}{d t}(\bar{X} Y)=\bar{X} \dot{Y}+\dot{\bar{X}} Y .
$$

Substituting for the derivatives from (1) and (2) there is obtained

$$
\begin{aligned}
\frac{d}{d t}\left(x_{i} y_{i}\right) & =\bar{X}(A Y+F)-(\overline{\bar{A} X}) Y \\
& =\bar{X} A Y+\bar{X} F-(\bar{X} A) Y .
\end{aligned}
$$

The first and last terms cancel identically and the result (3) follows.

Integrating (3) over the interval $[0, T]$, it is found that

$$
x_{i}(T) y_{i}(T)-x_{i}(0) y_{i}(0)=\int_{0}^{T} x_{i}(t) f_{i}(t) d t .
$$

This fundamental result is the one-dimensional form of Green's theorem.

The aim is now to calculate $y_{r+1}(0) \cdots y_{n}(0)$ using the given conditions. The method described in this section will utilize the adjoint system (2), whereas in the next section the original equations (1), only, will be used.

The adjoint system (2) must be integrated $(n-r)$ times from $T$ to 0 subject to certain starting values. These are chosen such that the $x_{i}(T)$ 's are all zero except one. For the $m^{\text {th }}$ integration this exception is the $x_{i}$ which appears as the coefficient of $y_{i_{m}}(T)$ in (4), and which may be designated by $x_{i_{m}}(T)$. For simplicity $x_{i_{m}}(T)$ is chosen to be unity.

Let ${ }_{m} x_{i}(t)$ be the value of $x_{i}$ deduced from the $m^{\text {th }}$ integration of the adjoint system. The fundamental result (4) holds for each integration of the adjoint system, and this is expressed by rewriting it

$$
{ }_{m} x_{i}(T) y_{i}(T)-{ }_{m} x_{i}(0) y_{i}(0)=\int_{0}^{T}{ }_{m} x_{i}(t) f_{i}(t) d t .
$$

But since all $x_{i}(T)$ 's were chosen zero except $x_{i_{m}}(T)$, the first summation reduces to a single term and (5) becomes

$$
{ }_{m} x_{i}(0) y_{i}(0)-y_{i_{m}}(T)=\int_{T}^{0}{ }_{m} x_{i}(t) f_{i}(t) d t .
$$


This is a system of $(n-r)$ linear algebraic equations for the $(n-r)$ unknowns $y_{i}(0), i=r+1 \cdots n$.

With these values determined (1) can now be integrated from 0 to $T$, as an initial value problem. The total number of integrations consists of $(n-r)$ of the adjoint system plus one of the original given system.

3. Linear Systems-Method of Complementary Functions. In this case the homogeneous equation, derived from (1),

$$
\dot{u}=A(t) u
$$

is integrated from 0 to $T(n-r)$ times. The $m^{\text {th }}$ time the initial conditions are

$$
\begin{array}{rlrl}
{ }_{m} u_{i}(0) & =0 & i \neq r+m \\
& =1 & i=r+m,
\end{array}
$$

where ${ }_{m} u_{i}(t)$ is the result of the $m^{\text {th }}$ integration of $u_{i}(t)$.

Next the non-homogeneous equation

$$
\dot{V}=A(t) V+F(t)
$$

is integrated once, from 0 to $T$, subject to

$$
\begin{aligned}
v_{i}(0) & =y_{i}(0) & & i=1 \cdots r \\
& =0 & & i=r+1 \cdots n .
\end{aligned}
$$

The general solution of (1), subject to the given conditions can be written

$$
y_{i}(t)=C_{p p} u_{i}(t)+v_{i}(t)
$$

where the $C_{p}$ 's are constants determined from the $(n-r)$ equations

$$
y_{i_{k}}(T)=C_{p p} u_{i_{k}}(T)+v_{i_{k}}(T) \quad k=1 \cdots(n-r) .
$$

In this equation the left-hand side is given and the quantities $u_{i}(T)$ and $v_{i}(T)$ have been determined from the integrations of (7) and (8). By letting $t=0$ in (9) it is seen that $C_{p}=y_{r+p}(0), p=1 \cdots n-r$.

The method of complementary functions takes less time than the method of adjoint equations for a single problem, but it is not so suitable when solutions are required for a large number of different forcing functions, $F$, or for a large number of different boundary conditions.

4. Nonlinear Systems. A general system of nonlinear, ordinary, differential equations may be written

$$
\dot{y}_{i}(t)=g_{i}\left(y_{1} \cdots y_{n}, t\right) \quad i=1 \cdots n,
$$


where it is assumed that the functions $g_{i}$ are once differentiable with respect to all the $y_{i}^{\prime}$ 's. Once again the boundary conditions are specified as $y_{1}(0) \cdots y_{r}(0)$ and $y_{i_{k}}(T), k=1 \cdots(n-r)$. The procedure is to estimate $y_{r+1}(0) \cdots y_{n}(0)$ which estimates are denoted by $y^{*}{ }_{r+1}(0) \cdots y_{n}{ }^{*}(0)$. Equations (11) can now be integrated as an initial value problem, and the solutions are denoted by $y_{i}{ }^{*}(t)$. In general, it will be found that the computed values of $y_{i_{k}}(T), k=1 \cdots(n-r)$ namely, $y^{*}{ }_{i_{k}}(T)$, differ from the given values of $y_{i_{k}}(T)$. To obtain the correct solution of the system (11) the difference between $y_{i_{k}}(T)$ and the given $y_{i_{k}}(T)$ must be made as small as possible.

Define $\delta y_{i}(t)$ by

$$
\delta y_{i}(t)=y_{i}(t)-y_{i}^{*}(t) \quad i=1 \cdots n .
$$

Substituting $y_{i}(t)$ in (11) there results, to a first approximation,

$$
\delta \dot{y}_{i}(t)=\left(\frac{\partial g_{i}}{\partial y_{j}}\right) \delta y_{j} .
$$

The equations adjoint to (13) are

$$
-\dot{x}_{i}=\left(\frac{\partial g_{j}}{\partial y_{i}}\right) x_{j}
$$

Since non-homogeneous terms are absent from (13) the Green's theorem for (13) and (14) reduces to

$$
x_{i}(T) \delta y_{i}(T)-x_{i}(0) \delta y_{i}(0)=0 .
$$

The quantities required are $\delta y_{i}(0), i=r+1 \cdots n$. To obtain $(n-r)$ equations, for these $(n-r)$ unknowns, (14) is integrated from $T$ to $0(n-r)$ times; the result of the $m^{\text {th }}$ integration is denoted by ${ }_{m} x_{i}(t)$. Equation (15) holds for each integration of the adjoint system, and this may be expressed by rewriting it

$$
{ }_{m} x_{i}(T) \delta y_{i}(T)-{ }_{m} x_{i}(0) \delta y_{i}(0)=0 \quad m=1 \cdots(n-r) .
$$

The $m^{\text {th }}$ time the adjoint equations are integrated the starting values are that all $x_{i}(T)$ 's are zero except the coefficient of $\delta y_{i_{m}}(T)$ which is chosen to be unity. The first summation in (16) now reduces to a single term; furthermore, $\delta y_{i}(0)$, $i=1 \cdots r$ are identically zero. Hence (16) reduces to

$$
\delta y_{i_{m}}(T)-{ }_{m} x_{r+i}(0) \delta y_{r+i}(0)=0 \quad m=1 \cdots(n-r),
$$

where the summation on $i$ extends from 1 to $(n-r)$. Substituting these values into (12) the improved estimate of $y_{i}(0), i=r+1 \cdots n$ are obtained. In the case of nonlinear systems (11) is integrated again, using the improved estimates, and the process is repeated. In the special case where the $g_{i}$ 's are linear functions 
of $y_{i}(13)$ is exact and the new estimates of the initial values are precise after only one application of the method. This method, when applied to linear systems, is similar to the second method described by Tifford [1].

5. Remarks. The problem was posed such that the $y_{i}$ 's were given at either end of the interval $0-T$. The methods are easily extended, if instead, $n-p$ linear relations are prescribed between the $y_{i}(0)$ 's and $p$ between the $y_{i}(T)$ 's. The methods may also be generalized to convert three or more point boundary value problems into an initial value problem.

An alternative method, suitable for nonlinear systems, has been presented by Lance and Deland [4]. This method is also one of iteration but it involves numerical differentiation and for this reason is less accurate per iteration than the method presented in section 4 .

If large scale computing machines are not available then relaxation methods can be used to solve systems of differential equations. Allen [5] states that if relaxation methods are to be used then an equal number of boundary conditions must be specified at each end of the interval, and for this reason the number of equations, $n$, must be even. Allen presents a method for converting problems which are not stated in this form into this form. The authors considered using the methods given above as an alternative to Allen's. Although, it is possible to do this, Reid [6] has shown that the conversion is unnecessary and that relaxation can be used directly regardless of how the boundary conditions are distributed. Reid also shows that the number of equations need not be even.

Cornell Aeronautical Laboratory, Inc.

T. R. Goodman

Buffalo, New York

Present address:

Allied Research Associates, Inc.,

Boston, Massachusetts

University of Southampton

G. N. LANCE

Southampton, England

The authors wish to thank Mr. A. H. Flax who suggested the basic idea of section 3 .

1. A. N. TIFford, "On the solution of total differential, boundary value problems," J. Aero. Sci., v. 18,1951 , p. $65-66$.

2. Gilbert Ames Bliss, Mathematics for Exterior Ballistics, John Wiley, New York, 1944, p. 63-71.

3. F. B. WRIGHT, JR., "The adjoint method in analog computation," Advisory Board on Simulation, Tech. Note 48, Univ. of Chicago, Illinois, 1954.

4. G. N. LANCE \& E. C. DELAND, "The steady axially symmetric flow of a viscous fluid in a deep rotating cylinder which is heated from below," Inst. Heat Transf. and Fluid Mech., Proc., v. $16,1955$.

5. D. N. DE G. Allen, Relaxation Methods, McGraw-Hill, New York, 1954, p. 218-225.

6. W. P. REID, "Relaxational solutions of non-jury-type differential equations," NAVORD Report 1963, NOTS 531, 1952. 